\section{QTc Prolongation and Torsades de Pointes in an Elderly Woman Taking Fluoxetine}

TO THE EDITOR: We describe an 83-year-old woman who experienced multiple episodes of torsades de pointes, probably because she was taking fluoxetine.

Transient unexplained loss of conscience in Ms. A led to her admission. Routine physical and laboratory examinations revealed no abnormalities (e.g., ischemia, electrolyte disturbances) besides a left bundle branch block and a prolonged QTc interval (478 $\mathrm{msec}$, corrected for QRS interval widening because of the left bundle branch block; reference $<450 \mathbf{~ m s e c}$ ). Her medications at admission were acetylsalicylic acid, $30 \mathrm{mg} /$ day, and fluoxetine, $20 \mathrm{mg} /$ day (serum levels at admission: fluoxetine, $204 \mu \mathrm{g} / \mathrm{liter}$; norfluoxetine, $138 \mu \mathrm{g} /$ liter); fluoxetine had been started 6 months earlier. During the night, continuous ECG recordings revealed recurrent short episodes of torsades de pointes. Multiple syncopal episodes occurred before admission, each time resolving spontaneously, which had never been observed before the initiation of fluoxetine.

Ms. A started having symptoms after the initiation of fluoxetine treatment and the documented pause-dependent polymorphic ventricular tachycardia characteristic of drug-induced QTc prolongation (1), which render fluoxetine use the most likely cause. Therefore, fluoxetine was discontinued. ECG recordings 2 and 8 months after fluoxetine discontinuation were normal apart from the left bundle branch block (QTc interval, $421 \mathrm{msec}$ and 408 msec, respectively, corrected for the widening of the QRS interval). No further episodes of syncope or tachyarrhythmias were seen.

ECG recordings in fluoxetine preregistration trials $(\mathrm{N}=312)$ showed no heart-block-inducing ECG conductance disturbances. However, four case reports implicating fluoxetine in QTc prolongation $(2,3)$ or torsades de pointes $(4,5)$ have been published. One involved intentional overdose (5), and one involved concomitant verapamil treatment (3). Because of fluoxetine's widespread use, the absence of cardiac conductance disturbances in the limited relatively healthy population in the preregistration phase is not very informative. However, at postregistration, thousands of ECG recordings in patients taking fluoxetine have been performed; in two studies focused on those with heart disease, no conduction disturbances were recorded. In our patient, there was a suggestive temporal relationship between the use of fluoxetine and the occurrence of QT prolongation and repeated episodes of torsades de pointes. After withdrawal of fluoxetine, the QTc interval returned to normal. In the absence of other plausible explanations, we conclude that the use of fluoxetine was the probable cause of the development of recurrent torsades de pointes. Older age, preexistent left bundle branch block, and female gender were likely additional risk factors (1).

Prescribers should be aware of the potential capacity of fluoxetine to prolong the QTc interval. Although it is probably extremely rare, it can have serious consequences for the patients involved.

\section{References}

1. Roden DM: Drug-induced prolongation of the QT interval. N Engl J Med 2004; 350:1013-1022
2. Ravina T, Suarez ML, Mendez-Castrillon J: Fluoxetine-induced QTU interval prolongation, T wave alternans and syncope. Int J Cardiol 1998; 65:311-313

3. Varriale P: Fluoxetine (Prozac) as a cause of QT prolongation. Arch Intern Med 2001; 161:612

4. Appleby M, Mbewu A, Clarke B: Fluoxetine and ventricular torsades-is there a link? Int J Cardiol 1995; 49:178-180

5. Lherm T, Lottin F, Larbi D, Bray M, Legall C, Caen D: [Torsades de pointes after poisoning with fluoxetine alone] (letter). Presse Med 2000; 29:306-307 (French)

INGEBORG WILTING, PHARM.D. O.M. SMALS, M.D. N.J. HOLWERDA, M.D. Tilburg, the Netherlands R.H. MEYBOOM, M.D., PH.D. M.L. De BRUIN, Pharm.D., Ph.D. T.C.G. EGBERTS, PHARM.D., PH.D. Utrecht, the Netherlands

\section{Pisa Syndrome in a Patient in a Wheelchair Taking Valproic Acid}

To THE EDITOR: Pisa syndrome (pleurothotonus) is a condition characterized by sustained flexion of the body with the head to one side, creating a "Leaning Tower" posture. It was first described by Ekbom et al. in 1972 (1). General incidence rates are not well established, although it appears to be more common in older patients; an $8 \%$ incidence was found in one series of newly admitted geropsychiatric patients (2). Most authors have described Pisa syndrome as a side effect of prolonged exposure to conventional neuroleptics (3). More recently, other agents, including atypical antipsychotics, cholinesterase inhibitors, antiemetics, and tricyclic antidepressants, have been implicated (4). Although treatment with anticholinergic agents has been proposed, definitive therapy remains the discontinuation of the offending agent (5). We report a case of suspected Pisa syndrome in a 65-year-old patient in a wheelchair receiving valproic acid.

Mr. A was a 65-year-old nursing home resident being treated for schizoaffective disorder. He was in a wheelchair secondary to severe arthritis. His medications were $750 \mathrm{mg}$ b.i.d. of valproic acid, $200 \mathrm{mg}$ b.i.d. of carbamazepine, and $3 \mathrm{mg}$ b.i.d. of risperidone-a regimen that had been stable and unchanged over several months. His valproic acid and carbamazepine levels were monitored routinely and were never above the therapeutic range.

Over a few weeks, the staff noticed that Mr. A had begun leaning to one side, a change that progressed to the point at which he was tilted at a $30^{\circ}$ angle throughout the day. He seemed unaware of and unconcerned by this change. He showed no other signs or symptoms suggestive of an extrapyramidal syndrome. The only medication change to which Mr. A consented was a trial discontinuation of valproic acid. Immediately upon discontinuation, his posture returned to a stable, upright position.

Pisa syndrome has not been reported in association with valproic acid, but our patient's dramatic improvement with its discontinuation strongly suggests a medication-related effect, either solely due to valproic acid or perhaps secondary to a pharmacodynamic interaction between valproic acid and risperidone. We believe that this patient's wheelchair state contributed to a delay in recognizing this condition and hope 